\title{
Neuroregulation News from Other Journals
}

\author{
Nancy L. Wigton ${ }^{1,2^{*}}$, Executive Editor \\ ${ }^{1}$ Grand Canyon University, Phoenix, Arizona, USA \\ ${ }^{2}$ Applied Neurotherapy Center, Scottsdale, Arizona, USA
}

\begin{abstract}
In the following section selected articles related to neuroregulation modalities are summarized. While most have a focus on EEG-based neurofeedback, some come from the growing fMRI-based neurofeedback approaches as well as transcranial magnetic stimulation neuromodulation. Of particular note, is the increase in prevalence of offerings from open access journals; many included here are from such journals, with direct URL links to the articles. The timeframe of these articles encompass from October 2013 through December 2014.
\end{abstract}

Citation: Wigton, N. L. (2015). Neuroregulation news from other journals. NeuroRegulation, 2(1), 52-56. doi.org/10.15540/nr.2.1.52

*Address correspondence to: Nancy Wigton, PhD, Applied Edited by:

Neurotherapy Center, 10200 N. 92nd Street, Suite 120, Scottsdale, AZ Rex Cannon, PhD, Positive Brain Training, Florida, USA

85258, USA. Email: Dr.Wigton@appliedneurotherapy.com

Copyright: ( 2 2015. ISNR. This is an Open Access article distributed under the terms of the Creative Commons Attribution License (CCBY).

\section{Applied Psychophysiology and Biofeedback}

Escolano, C., Navarro-Gil, M., Garcia-Campayo, J., Congedo, M., \& Minguez, J. (2014). The effects of individual upper alpha neurofeedback in ADHD: An open-label pilot study. Applied Psychophysiology and Biofeedback, 39(3-4), 193-202. http://dx.doi.org/10.1007/s10484-0149257-6

This study of 20 children with ADHD, evaluated an individualized approach to neurofeedback by incorporating alpha peak frequency in the protocol development. Neurophysiological tests, outcome measures such as parent rating scales, and assessed learning curves were included to evaluate efficacy.

Sokhadze, E. M., El-Baz, A. S., Tasman, A., Sears, L. L., Wang, Y., Lamina, E. V., \& Casanova, M. F. (2014). Neuromodulation integrating rTMS and neurofeedback for the treatment of autism spectrum disorder: An exploratory study. Applied Psychophysiology and Biofeedback, 39(3-4), 237-257. http://dx.doi.org/10.1007/s10484-014$\underline{9264-7}$

This study investigated the effects of repetitive transcranial magnetic stimulation (rTMS) in combination with EEG neurofeedback (TMS-NF) as compared to a waitlist control group for the treatment of Autism spectrum disorder. The study found improved behavioral and function outcomes from the 18 sessions of TMS-NF, more so over the waitlist control group.

Steiner, N. J., Frenette, E., Hynes, C., Pisarik, E., Tomasetti, K., Perrin, E. C., \& Rene, K. (2014). A pilot feasibility study of neurofeedback for children with autism. Applied Psychophysiology and Biofeedback, 39(2), 99-107. http://dx.doi.org/10.1007/s10484-014-9241-1

This was a study focusing on neurofeedback for children with high functioning autism spectrum disorders and difficulties with attention. It examines the feasibility of neurofeedback with this population.

\section{Biofeedback}

Koberda, J. L., Koberda, P., Moses, A., Winslow, J., Bienkiewicz, A., \& Koberda, L. (2014). Z-score LORETA neurofeedback as a potential therapy for ADHD. Biofeedback, 42(2), 74-81. http://dx.doi.org/10.5298/1081-5937-42.2.05 
This is a single case study report of the use of both surface and low-resolution brain electromagnetic tomography (LORETA) z-score neurofeedback with an 8-year-old male with ADHD. Cognitive testing demonstrated improvements in symptoms as a result of the $z$-score neurofeedback. There were also improvements in academic functioning.

Thatcher, R. W. (2014). Advances in assessment and treatment of ADHD using network analyses. Biofeedback, 42(2), 58-67.

http://dx.doi.org/10.5298/1081-5937-42.2.07

A review article discussing z-score neurofeedback, the article discusses the benefits of $z$-score neurofeedback derived from the neuroscience underpinnings of brain networks. Specific networks (such as attention or default mode) can be directly addressed through the implementation of LORETA $z$-score neurofeedback; thus allowing improved specificity in the linking of symptoms with brain dysregulation.

\section{Biological Psychology}

In January 2014, the Biological Psychology journal published a special themed issue where all articles in the volume focused on neurofeedback. Included in that issue were 13 articles that encompassed a wide variety of topics within the field of neurofeedback. The titles of those articles are as follows, and all are found in Volume 95:

Arns, Heinrich, and Strehl. Evaluation of neurofeedback in ADHD: The long and winding road.

http://dx.doi.org/10.1016/j.biopsycho.2013.11.013

Dekker, Sitskoorn, Denissen, and van Boxtel. The time-course of alpha neurofeedback training effects in healthy participants.

http://dx.doi.org/10.1016/j.biopsycho.2013.11.014

Enriquez-Geppert et al. Modulation of frontal-midline theta by neurofeedback. http://dx.doi.org/10.1016/j.biopsycho.2013.02.019

Gruzelier, Foks, Steffert, Chen, and Ros. Beneficial outcome from EEG-neurofeedback on creative music performance, attention and well-being in school children.

http://dx.doi.org/10.1016/j.biopsycho.2013.04.005

Gruzelier et al. Replication of elite music performance enhancement following alpha/theta neurofeedback and application to novice performance and improvisation with SMR benefits.

http://dx.doi.org/10.1016/j.biopsycho.2013.11.001

Kober et al. Near-infrared spectroscopy based neurofeedback training increases specific motor imagery related cortical activation compared to sham feedback.

http://dx.doi.org/10.1016/j.biopsycho.2013.05.005

Maurizio et al. Comparing tomographic EEG neurofeedback and EMG biofeedback in children with attention-deficit/hyperactivity disorder. http://dx.doi.org/10.1016/j.biopsycho.2013.10.008

Meisel, Servera, Garcia-Banda, Cardo, and Moreno. Reprint of "Neurofeedback and standard pharmacological intervention in ADHD: A randomized controlled trial with six-month followup."

http://dx.doi.org/10.1016/j.biopsycho.2013.09.009

Reiner, Rozengurt, and Barnea. Better than sleep: Theta neurofeedback training accelerates memory consolidation.

http://dx.doi.org/10.1016/j.biopsycho.2013.10.010

Ros, Munneke, Parkinson, and Gruzelier. Neurofeedback facilitation of implicit motor learning. http://dx.doi.org/10.1016/j.biopsycho.2013.04.013

Ruiz, Buyukturkoglu, Rana, Birbaumer, and Sitaram. Real-time fMRI brain computer interfaces: Selfregulation of single brain regions to networks. http://dx.doi.org/10.1016/j.biopsycho.2013.04.010

Schabus et al. Enhancing sleep quality and memory in insomnia using instrumental sensorimotor rhythm conditioning. http://dx.doi.org/10.1016/j.biopsycho.2013.02.020

Staufenbiel, Brouwer, Keizer, and van Wouwe. Effect of beta and gamma neurofeedback on memory and intelligence in the elderly. http://dx.doi.org/10.1016/j.biopsycho.2013.05.020

\section{Child and Adolescent Psychiatric Clinics of North America}

Holtmann, M., Sonuga-Barke, E., Cortese, S., \& Brandeis, D. (2014). Neurofeedback for ADHD: A review of current evidence. Child and Adolescent Psychiatric Clinics of North America, 23(4), 789806. http://dx.doi.org/10.1016/j.chc.2014.05.006

This article is an overall review of neurofeedback. The authors call for more studies, which are methodologically sound, well controlled, and include larger samples. However, there is acknowledgement that neurofeedback has gained empirical support more recently and fits within in multimodal treatment therapy approaches. 


\section{Clinical EEG and Neuroscience}

Cannon, R. L., Baldwin, D. R., Diloreto, D. J., Phillips, S. T., Shaw, T. L., \& Levy, J. J. (2014). LORETA neurofeedback in the precuneus: Operant conditioning in basic mechanisms of self-regulation. Clinical EEG and Neuroscience, 45(4), 238-248.

http://dx.doi.org/10.1177/1550059413512796

A research study evaluating low-resolution brain electromagnetic tomography (LORETA) neurofeedback in the precuneus with 13 participants (five non-clinical, eight with heterogeneous clinical diagnoses). Outcome measures in the form of neurophysiological data, a personality inventory, and an executive function rating scale were assessed to determine the effects of the neurofeedback. This study provides support for the use of LORETA neurofeedback for enhancement of executive function and improvement in psychological symptoms.

\section{Clinical Neurophysiology}

Mottaz, A., Solcà, M., Magnin, C., Corbet, T., Schnider, A., \& Guggisberg, A. G. (2014). Neurofeedback training of alpha-band coherence enhances motor performance. Clinical Neurophysiology. Advance online publication. http://dx.doi.org/10.1016/j.clinph.2014.11.023

This is a research article evaluating neurofeedback to address functional connectivity. The sample included one stroke patient and 10 healthy subjects. The frequency of interest was the alpha band.

\section{Frontiers in Human Neuroscience}

Arns, M., Feddema, I., \& Kenemans, J. L. (2014). Differential effects of theta/beta and SMR neurofeedback in ADHD on sleep onset latency. Frontiers in Human Neuroscience, 8(1019), 1-10. http://dx.doi.org/10.3389/fnhum.2014.01019

This is a research study evaluating the role of sensory motor rhythm (SMR) neurofeedback versus theta beta ratio neurofeedback and the impact of sleep and attention-deficit/hyperactivity disorder (ADHD). The study compared adult ADHD patients to control group participants. Relationships were found between inattention in adults and self-reported sleep problems. The SMR neurofeedback resulted in more improvement in sleep symptoms. Possible differential effects of SMR and theta beta ratio neurofeedback are discussed.

Gevensleben, H., Moll, G. H., Rothenberger, A., \& Heinrich, H. (2014). Neurofeedback in attentiondeficit/hyperactivity disorder - different models, different ways of application. Frontiers in Human Neuroscience, 8(846), 1-10.

http://dx.doi.org/10.3389/fnhum.2014.00846

A review article discussing two hypothetical neurofeedback framework models informed by either slow cortical potential or frequency band neurofeedback modalities. The authors suggest the underlying model impacts how the neurofeedback is evaluated and applied. They argue the importance of stating theoretical perspectives of frameworks and models when reporting on neurofeedback research.

Kober, S. E., Witte, M., Ninaus, M., Neuper, C., \& Wood, G. (2013). Learning to modulate one's own brain activity: The effect of spontaneous mental strategies. Frontiers in Human Neuroscience, 7(695), 1-12.

http://dx.doi.org/10.3389/fnhum.2013.00695

This research compared 10 neurofeedback sessions of either sensorimotor rhythm based or gamma (40$43 \mathrm{~Hz}$ ) based protocols. Also investigated were mental strategies on neurofeedback performance. Participants who reported having no specific mental strategy demonstrated linear improvements in neurofeedback performance whereas participants reporting specific mental strategies did not.

Ros, T., Baars, B. J., Lanius, R. A., \& Vuilleumier, P. (2014). Tuning pathological brain oscillations with neurofeedback: A systems neuroscience framework. Frontiers in Human Neuroscience, 8(1008), 1-22.

http://dx.doi.org/10.3389/fnhum.2014.01008

This paper is a review article providing a broad overview of the neurophysiological underpinnings of neurofeedback. Also discusses a proposed mechanism for the workings of neurofeedback in terms of tuning brain oscillations in a framework of network stability and flexibility. 
Strehl, U. (2014). What learning theories can teach us in designing neurofeedback treatments. Frontiers in Human Neuroscience, 8(894), 1-8. http://dx.doi.org/10.3389/fnhum.2014.00894

A review article exploring various aspects of learning theory as applied to neurofeedback. The author also provides a reminder of the importance of psychotherapeutic dynamics in the neurofeedback process.

Strehl, U., Birkle, S. M., Wörz, S., \& Kotchoubey, B. (2014). Sustained reduction of seizures in patients with intractable epilepsy after selfregulation training of slow cortical potentials -10 years after. Frontiers in Human Neuroscience, 8(604), 1-7.

http://dx.doi.org/10.3389/fnhum.2014.00604

This article is a long-term follow-up study of slow cortical potential (SCP) neurofeedback for intractable epilepsy. The study was conducted 10 years after the neurofeedback and is reported to be the longest follow-up study for psychophysiological epilepsy treatment. The study suggests participants were still able to self-regulate their SCPs and continued to experience reduced frequency of seizures.

\section{Human Brain Mapping}

Zhang, G., Yao, L., Shen, J., Yang, Y., \& Zhao, X. (2014). Reorganization of functional brain networks mediates the improvement of cognitive performance following real-time neurofeedback training of working memory. Human Brain Mapping. Advance online publication.

http://dx.doi.org/10.1002/hbm.22731

This real-time fMRI neurofeedback study investigated connectivity between the working memory network and default mode network. Results suggest training on the identified regions of interest improved cognitive performance.

\section{Memory}

Guez, J., Rogel, A., Getter, N., Keha, E., Cohen, T., Amor, T., ... Todder, D. (2014). Influence of electroencephalography neurofeedback training on episodic memory: A randomized, sham- controlled, double-blind study. Memory. Advance online publication.

http://dx.doi.org/10.1080/09658211.2014.921713

A research study evaluating neurofeedback effects on memory using a double-blind sham-controlled design. The study participants included 30 healthy adults who engaged in 10 neurofeedback sessions. Results of pretest and posttest memory assessment are reported.

\section{Neurolmage: Clinical}

Stoeckel, L. E., Garrison, K. A., Ghosh, S. S., Wighton, P., Hanlon, C. A., Gilman, J. M., ... Evins, A. E. (2014). Optimizing real time fMRI neurofeedback for therapeutic discovery and development. Neurolmage: Clinical, 5, 245-55. http://dx.doi.org/10.1016/j.nicl.2014.07.002

This paper is a review article discussing real time functional magnetic resonance imaging (rtfMRI) neurofeedback applications. The authors propose guidelines for rtfMRI studies and call for clinical trials of this modality. They believe that rtfMRI neurofeedback can be a personalized intervention approach to address maladaptive brain patterns.

\section{NeuroRehabilitation}

Munivenkatappa, A., Rajeswaran, J., Indira Devi, B., Bennet, N., \& Upadhyay, N. (2014). EEG neurofeedback therapy: Can it attenuate brain changes in TBI? NeuroRehabilitation, 35(3), 481484. http://dx.doi.org/10.3233/NRE-141140

This is a case review study of two individuals with histories of traumatic brain injury. Both participants received 20 neurofeedback sessions over a period of 2 months. Cognitive functions for both individuals were improved upon post testing.

\section{Neuroscience and Biobehavioral Reviews}

Gruzelier, J. H. (2014). EEG-neurofeedback for optimising performance. I: A review of cognitive and affective outcome in healthy participants. Neuroscience and Biobehavioral Reviews, 44, 124-141.

http://dx.doi.org/10.1016/j.neubiorev.2013.09.015 
Gruzelier, J. H. (2014). EEG-neurofeedback for optimising performance. II: Creativity, the performing arts and ecological validity. Neuroscience and Biobehavioral Reviews, 44, 142-158.

http://dx.doi.org/10.1016/j.neubiorev.2013.11.004

Gruzelier, J. H. (2014). EEG-neurofeedback for optimising performance. III: A review of methodological and theoretical considerations. Neuroscience and Biobehavioral Reviews, 44, 159-182.

http://dx.doi.org/10.1016/j.neubiorev.2014.03.015

A three-part series of review articles discussing neurofeedback from the perspective of optimizing performance. Part one is an overview of the neurofeedback field in general and reviews studies evaluating healthy and elderly participants. Of those reviewed, the authors indicate 23 controlled studies report outcomes that are beneficial. Part two primarily focuses on the use of neurofeedback for enhancement of creativity in the performing arts, to include acting, music, and dance. Part three is germane to researchers and practitioners in discussing theoretical and methodological issues as it pertains to the scientific study and practice of neurofeedback.

\section{Pediatrics}

Steiner, N. J., Frenette, E. C., Rene, K. M., Brennan, R. T., \& Perrin, E. C. (2014). In-school neurofeedback training for ADHD: Sustained improvements from a randomized control trial. Pediatrics, 133(3), 483-492.

http://dx.doi.org/10.1542/peds.2013-2059

This randomized controlled study evaluated sustained gains from 40 sessions of neurofeedback, cognitive training, or a control condition, 6 months after the training. The sample included 104 children ages 7- to 11-years-old who were all diagnosed with attention-deficit/hyperactivity disorder (ADHD). Outcome measures were three behavioral rating scales and changes in medication dosages. Greater improvements in ADHD symptoms were attained by the neurofeedback group over the cognitive training or control group; moreover, these gains were sustained at the 6-month follow-up. Also of note was that, at the 6-month follow-up, both the cognitive training and control groups had increases (statistically and clinically) in medication, while only the neurofeedback group maintained the same dosage levels.

\section{PLoS ONE}

Neuner, I., Arrubla, J., Werner, C. J., Hitz, K., Boers, F., Kawohl, W., \& Shah, N. J. (2014). The default mode network and EEG regional spectral power: A simultaneous fMRI-EEG study. PLOS ONE, 9(2), e88214, 1-8.

http://dx.doi.org/10.1371/journal.pone.0088214

This is a study evaluating fMRI and EEG data simultaneously as it relates to the default mode network. The EEG was investigated using a region of interest approach with Low Resolution Electromagnetic Tomography (LORETA). The results suggest support for EEG band specific associations with various default mode network functions.

\section{Psychology of Addictive Behaviors}

Keith, J. R., Rapgay, L., Theodore, D., Schwartz, J. M., \& Ross, J. L. (2014). An assessment of an automated EEG biofeedback system for attention deficits in a substance use disorders residential treatment setting. Psychology of Addictive Behaviors. Advance online publication. http://dx.doi.org/10.1037/adb0000016

This research study was conducted to evaluate the effectiveness of neurofeedback in a residential treatment setting for substance use. Here an automated neurofeedback protocol was compared to clinician-guided protocols, as well as to participants who received additional therapy sessions as a control condition. All groups received 15 sessions of each protocol or additional therapy and group sample sizes ranged from 30 to 33 . Both the neurofeedback groups resulted in post treatment improvements in the computerized performance test outcome measure, with no improvement for the control group. No significant difference between the automated or clinician-guided neurofeedback was found.

Received: February 1, 2015

Accepted: March 17, 2015

Published: April 14, 2015 